\title{
Effect of aging on formation of reactive oxygen species by mitochondria of rat heart
}

\author{
Stanislav Kuka, Zuzana Tatarkova, Peter Racay, Jan Lehotsky, Dusan Dobrota and Peter Kaplan \\ Department of Medical Biochemistry, Jessenius Faculty of Medicine, Comenius University, Martin, Slovak Republic
}

\begin{abstract}
Mitochondrial electron transport chain is thought to be a major source of reactive oxygen species (ROS) during aging. However, this view is supported mainly by accumulation of mitochondrial oxidative damage with age and the exact sites of ROS formation remains unknown. In the present study, we measured rate of ROS formation using 2',7'-dichlorofluorescein (DCF) probe in cardiac mitochondria from adult (6-month-old), old (15-month-old) and senescent (26-month-old) rats. In mitochondria oxidizing complex II substrate, succinate, the rate of ROS formation progressively increased with age. In the presence of complex I inhibitor rotenone or complex III inhibitor antimycin A, the rate ROS formation significantly decreased, but even the combination of inhibitors could not fully prevent generation of ROS. Age-dependent increase of ROS formation was accompanied by a loss of thiol groups, tryptophan degradation and increased lipid peroxidation. These data suggest that in addition to complex I and complex II other mitochondrial sites can contribute to accelerated ROS generation and oxidative damage during aging.
\end{abstract}

Key words: Aging - Heart - Mitochondria - ROS - Oxidative stress

\section{Introduction}

Aging of the heart is characterized by progressive alterations in structure and physiological function. Although the mechanisms underlying cardiac alterations are not fully understood, mitochondrial dysfunction is believed to play a key role in the aging process (Dai et al. 2012). According to mitochondrial oxidative stress theory of aging, reactive oxygen species (ROS) produced as by-products of mitochondrial bioenergetics accumulate with age and cause cellular oxidative damage resulting in tissue and organ dysfunction. It is generally accepted that mitochondria as a primary site of ROS production are also the major target of their damaging effects. Age-associated mutations of mitochondrial DNA and altered transcription of genes coding for proteins involved in oxidative phosphorylation were demonstrated in rat heart (Wanagat et al. 2002; Preston et al. 2008). Furthermore, deterioration of function of mitochondrial proteins with age as a consequence of posttranslational oxidative

Correspondence to: Peter Kaplan, Department of Medical Biochemistry, Jessenius Faculty of Medicine, Comenius University, Mala Hora 4, 03601 Martin, Slovak Republic

E-mail: kaplan@jfmed.uniba.sk modification was demonstrated in several studies (Choksi and Papaconstantinou 2008; Preston et al. 2008) and also in our laboratory (Tatarková et al. 2011). Protein modification in aged heart can also result from accumulation of products of lipid peroxidation (LPO). Reactive aldehydes, such as 4-hydroxynonenal and malondialdehyde, were shown to increase with age and to modify several proteins (Tatarková et al. 2011). In addition, LPO of cardiolipin, a phospholipid which occurs almost exclusively in mitochondrial membrane, decreases the activity of electron transport chain (ETC) complexes (Petrosillo et al. 2009). Decrease in activity ETC complexes with age has been reported in the number of studies (Tatarkova et al. 2011; Gómez and Hagen 2012; Hunter et al. 2012). Since ETC is known to be the major cellular source of ROS, oxidative damage of its complexes can further increase the leakage of ROS resulting in elevated oxidative stress. In contrast to ROS-induced oxidative damage, direct measurement of generation of short-lived ROS is difficult. Superoxide anion radical $\left(\mathrm{O}_{2}{ }^{-}\right)$, hydrogen peroxide or other reactive species were detected mainly by utilizing redox-sensitive fluorescent probes. However, these studies did not consistently support the age-dependent increase in ROS production (see Barja 1999; Gómez and Hagen 2012). While some studies have shown increased formation of ROS 
during aging (Sohal et al. 1994; Bejma et al. 2000; Moghaddas et al. 2003; Petrosilo et al. 2009) other suggests that ROS production is unchanged (Hansford et al. 1997). One of these studies attempted to identify ETC complexes involved in the release of $\mathrm{O}_{2}{ }^{-}$. The results suggest greater leak of ROS from complex III (Moghaddas et al. 2003). Sites of ROS formation in ETC have been investigated in many studies not related to aging. The results showed that complex III can produce $\mathrm{O}_{2} \cdot^{-}$, but to a much lower extend then complex I (see Barja 1999; Murphy 2009). Complex I produces $\mathrm{O}_{2}{ }^{-}$by two mechanisms, at high $\mathrm{NADH} / \mathrm{NAD}^{+}$ratio when flavin mononucleotide (FMN) site on complex I is reduced and by reverse electron transport (RET) due to high membrane potential and reduced coenzyme Q. It has been suggested that complex I is the major site of superoxide production in mitochondria (Murphy 2009).

Therefore, purpose of the present study was to determine the effect of aging on ROS formation in cardiac mitochondria and to evaluate the potential roles of ETC complexes in this process. Using redox-sensitive fluorescent probe dichlorodihydrofluorescein $\left(\mathrm{DCF}_{2}\right)$ and inhibitors of ETC complexes I and III we compared ROS production in mitochondria prepared from hearts of 6-, 15- and 26-monthold rats. Our results indicate that ROS formation increases during aging and may cause damage to mitochondrial lipids and proteins.

\section{Materials and Methods}

\section{Animals}

Fifteen male Wistar rats (supplied by Institute of Experimental Pharmacology, Slovak Academy of Sciences, Dobra Voda, Slovak Republic) were divided into three groups (5 rats per group) according to age, as adult (6-month-old), old (15month-old) and senescent (26-month-old). The animals were maintained in an air-conditioned room as described previously (Kaplan et al. 2007). Experiments were performed in accordance with the "Guide for the Care and Use of Laboratory Animals" published by The US National Institute of Health (NIH publication NO 85-23, revised 1996), and the ethical guidelines of the Jessenius Faculty of Medicine.

\section{Preparation of tissue homogenates and mitochondria}

The animals were decapitated after anesthetization by halothane (3\% halothane in oxygen/nitrous oxide, 1:2). After cannulation of the aorta, the hearts were immediately washed with physiological solution and stored at $-80^{\circ} \mathrm{C}$ until used. Frozen powdered tissue of the whole heart (about $1 \mathrm{~g}$ ) was thawed in 10 volumes of ice-cold homogenization buffer (30 mM KH $\mathrm{KH}_{4}, 5 \mathrm{mM}$ EDTA, $0.3 \mathrm{M}$ sucrose, $\mathrm{pH}$ 7.0) with $0.3 \mathrm{mM}$ phenylmethylsulfonyl fluoride (PMSF) and homogenized in Potter-Elvehjem homogenizer. Cardiac mitochondrial fraction was isolated from individual tissue homogenates by differential centrifugation as previously described (Babusikova et al. 2004).

\section{Measurement of mitochondrial ROS production}

The mitochondrial ROS production in stage 4 respiration was measured by a fluorimetric method using the probe 2',7'-dichlorofluorescein (DCF). The measurements were performed in solution containing $5 \mu \mathrm{g} / \mathrm{ml}$ mitochondrial proteins, $0.1 \mathrm{~mol} / 1 \mathrm{KH}_{2} \mathrm{PO}_{4}$ and $5 \mu \mathrm{mol} / 1 \mathrm{DCFH}_{2}$-DA using $2 \mathrm{mmol} / \mathrm{l}$ succinate as a substrate. The reaction was started by the addition of mitochondria in the presence and the abscence of complex I inhibitor rotenone $(10 \mu \mathrm{mol} / \mathrm{l})$ or complex III inhibitor antimycin A $(5 \mu \mathrm{mol} / \mathrm{l})$. Probe was added in form of membrane permeable dichlorodihydrofluorescein diacetate $\left(\mathrm{DCFH}_{2}-\mathrm{DA}\right)$. Rate of ROS production was estimated from the rate of oxidation of deacetylated $\mathrm{DCFH}_{2}$ to DCF following the slope of fluorescence increase at $520 \mathrm{~nm}$ (10 nm slit width) excited at $485 \mathrm{~nm}$ (5 nm slit width). The concentrations of DCF formed in mitochondria were determined from standard curve using known concentrations of DCF.

\section{Measurement of thiol group content}

Content of thiol groups in cardiac mitochondria (aliquots of $0.15 \mathrm{mg}$ proteins) was determined spectrophotometrically using DTNB (5,5'-dithiobis[2-nitrobenzoic acid]) as described previously (Tatarková et al. 2011). Samples were incubated in medium containing $30 \mathrm{mM}$ imidazole ( $\mathrm{pH}$ 7.4), $5 \mathrm{mM}$ EDTA, $0.4 \mathrm{mM}$ DTNB. The thiol group content was calculated from absorbances at $412 \mathrm{~nm}$ using molar absorption coefficient $\varepsilon=13,600 \mathrm{M}^{-1} \mathrm{~cm}^{-1}$.

\section{Measurement of tryptophan degradation}

The steady-state fluorescence measurements were performed in solutions containing $50 \mu \mathrm{g}$ of mitochondrial protein/ml, $10 \mathrm{mM} \mathrm{HEPES} \mathrm{(pH} \mathrm{7.0),} 100 \mathrm{mM} \mathrm{KCl}$ at $25^{\circ} \mathrm{C}$ on PerkinElmer LS-55 spectrofluorimeter. The fluorescence emission spectra (between $310-450 \mathrm{~nm}, 5 \mathrm{~nm}$ slit width) of tryptophan were measured by excitation at $295 \mathrm{~nm}(5 \mathrm{~nm}$ slit width).

\section{Measurement of lipid peroxidation}

Lipid peroxidation in cardiac mitochondria was measured according to the formation thiobarbituric acid reactive substances (TBARS) as described previously (Tatarkova et al. 2012). The concentration of TBARS was calculated according 


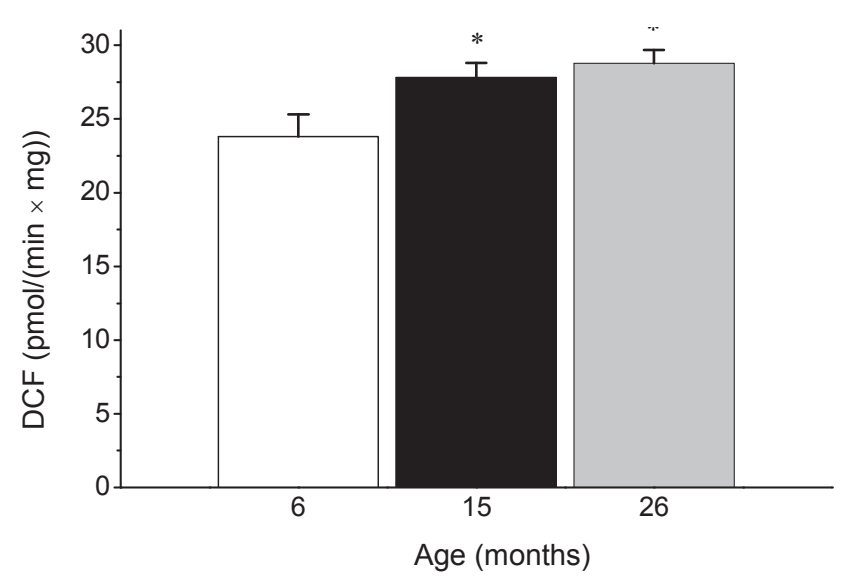

Figure 1. Effect of aging on ROS generation in cardiac mitochondria. ROS formation was measured by monitoring the fluorescence increase of oxidized DCF probe in isolated mitochondria supplemented with $2 \mathrm{mmol} / \mathrm{l}$ succinate as a substrate. To calculate the rate of DCF formation, the slope difference after addition of mitochondria was used. Values are given as means $\pm \operatorname{SEM}(n=5) .{ }^{\star} p<0.05$, significantly different when compared to 6 -month-old rats. There were no significant differences between 15- and 26-month-old rats. DCF, dichlorofluorescein.

to the absorbance measured at $535 \mathrm{~nm}$ using the molar extinction coefficient of malondialdehyde $\left(1.56 \times 10^{5} \mathrm{~mol} / \mathrm{l} / \mathrm{cm}\right)$.

\section{Statistical analysis}

Data are expressed as mean \pm SEM. One-way analysis of variance with post-hoc comparisons by Student-Neuman-Keuls test was carried out to test for differences among groups (GrafPhad software). A value of $p<0.05$ was considered to be statistically significant.

\section{Results}

The age-dependent changes of body weight, heart weight and heart-to-body weight ratios are shown in Table 1. Both, body weight and heart weight of old and senescent rats were significantly greater than those of adult rats. However, heart-to-body weight ratio, which is an indicator of cardiac hypertrophy, was unaltered.

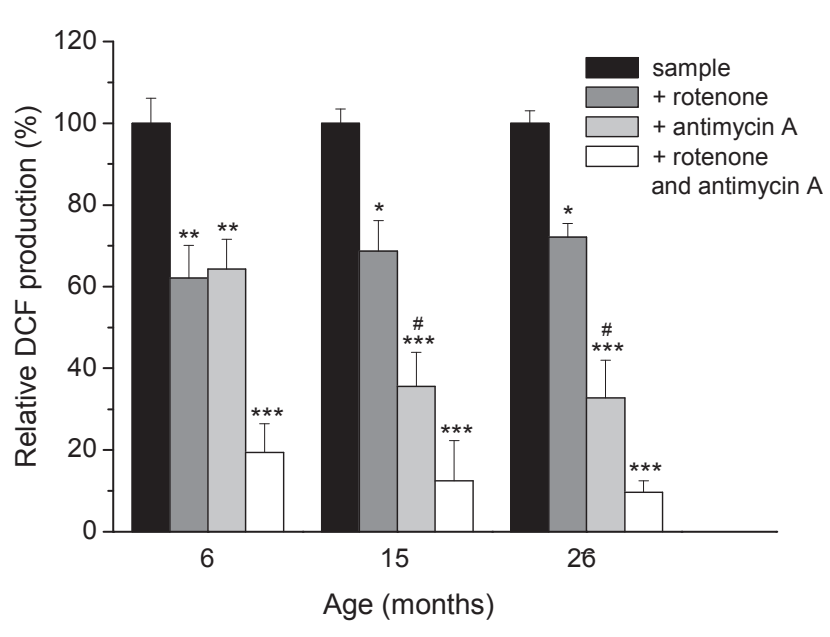

Figure 2. Effects of electron transport chain inhibitors on ROS generation in cardiac mitochondria of adult, old and senescent rats. Isolated mitochondria were supplemented with $2 \mathrm{mmol} / \mathrm{l}$ succinate, the inhibitor of complex I $(10 \mu \mathrm{mol} / \mathrm{l}$ rotenone $)$ and the inhibitor of complex III ( $5 \mu \mathrm{mol} / \mathrm{l}$ antimycin A ) were used alone or in combination. The data were normalized in respect to the ROS formation in the same age group in the absence of inhibitors (black bars). Values are given as means $\pm \operatorname{SEM}(n=5) .{ }^{*} p<0.05,{ }^{* *} p<0.01,{ }^{* *} p<0.001$, significantly different when compared to the same age group without inhibitors; ${ }^{\#} p<0.05$, significantly different from corresponding value in 6-month-old rats. There were no significant differences between 15- and 26-month-old rats. DCF, dichlorofluorescein.

To evaluate the role of aging in mitochondrial formation of ROS, we measured rate of conversion of $\mathrm{DCFH}_{2}$ to fluorescent DCF in cardiac mitochondria isolated from adult (6-month-old), old (15-month-old) and senescent (26month-old) rats. As shown in Fig. 1, the rate of ROS formation induced by succinate progressively increased with age, in old and senescent rat hearts the rate increased by $16.9 \pm 4.1 \%$ $(p<0.01)$ and $20.9 \pm 3.4 \%(p<0.01)$, respectively. The role of RET in the formation of ROS was evaluated by using the complex I inhibitor rotenone. As illustrated in Fig. 2, treatment of mitochondria with rotenone significantly reduced the rate of ROS formation in all age groups, but there were no significant differences in the rate decrease among the groups. In adult, old and senescent rats the DCF fluorescence rate decreased by $37.8 \pm 8.0(p<0.01), 31.3 \pm 7.5(p<0.05)$

Table 1. Effect of aging on body weight, heart weight and heart-to-body weight ratio

\begin{tabular}{lccc}
\hline Rats & Body weight $(\mathrm{g})$ & Heart weight $(\mathrm{g})$ & Heart/body weight $(\mathrm{mg} / \mathrm{g})$ \\
\hline 6-months-old & $289 \pm 6.7$ & $0.89 \pm 0.07$ & $3.09 \pm 0.18$ \\
15-months-old & $475 \pm 15.0^{* * *}$ & $1.34 \pm 0.05^{*}$ & $2.82 \pm 0.17$ \\
26-months-old & $480 \pm 11.3^{* * *}$ & $1.51 \pm 0.11^{* *}$ & $3.15 \pm 0.16$ \\
\hline
\end{tabular}

Values are expressed as means $\pm \operatorname{SEM}(n=5) .{ }^{*} p<0.05,{ }^{* *} p<0.01,{ }^{* * *} p<0.001$, significantly different as compared to 6-months-old rats. 

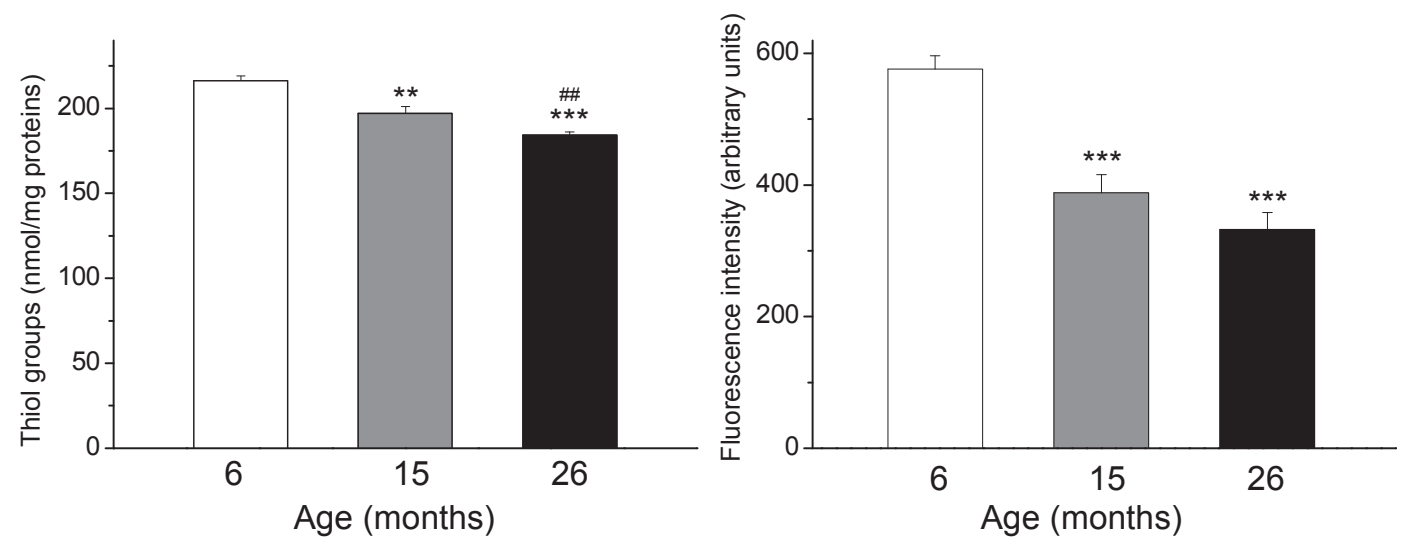

Figure 3. Effect of aging on oxidation of proteins in rat cardiac mitochondria. Protein oxidation was measured in isolated mitochondria by monitoring the loss of thiol groups using DTNB assay $(\mathbf{A})$ and by measuring decrease of fluorescence emission spectra between 310 $450 \mathrm{~nm}$ corresponding to tryptophan $(\mathbf{B})$. Values are given means $\pm \operatorname{SEM}(n=5) .{ }^{* *} p<0.01,{ }^{* * *} p<0.001$, significantly different when compared to 6-month-old rats; ${ }^{\# \#} p<0.01$, significantly different when compared to 15 -month-old rats.

and $27.9 \pm 2.5 \%(p<0.05)$, respectively. The rate of ROS formation was also studied in the presence of complex III inhibitor antimycin A. Treatment of mitochondria with this inhibitor also significantly reduced ROS formation, but the decrease was age-dependent (Fig. 2). In mitochondria of adult rats DCF fluorescence decreased by $35.7 \pm 10.9 \%$ $(p<0.01)$, whereas in old and senescent rats by $64.5 \pm 8.4 \%$ $(p<0.001)$ and $67.3 \pm 9.3 \%(p<0.001)$, respectively. In old and senescent rats the rotenone-induced decreases differ significantly from the corresponding value in adult rats $(p<0.05)$, but differences between these two groups were not significant. When mitochondria were treated with combination of rotenone and antimycin A the DCF formation

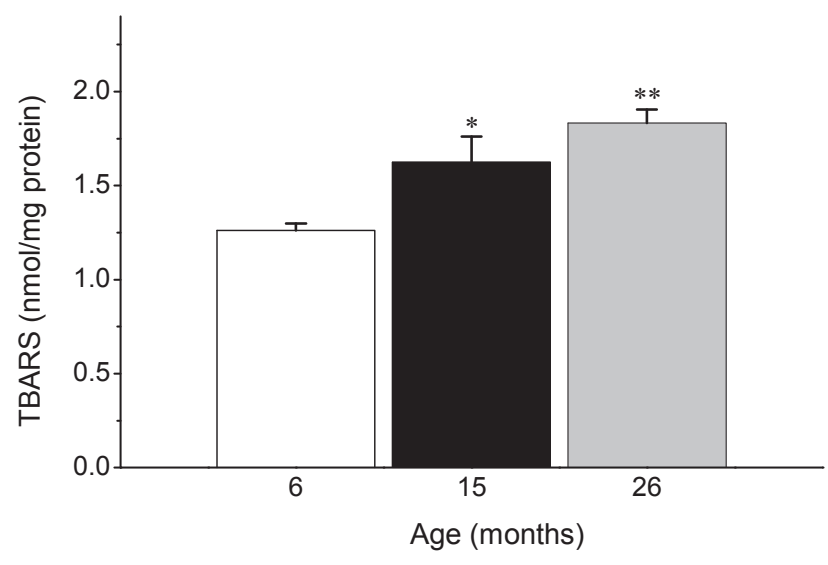

Figure 4. Effect of aging on lipid peroxidation in rat cardiac mitochondria. Lipid peroxidation was estimated from the content of thiobarbituric acid reactive substances (TBARS) in isolated mitochondria. Values are given means $\pm \operatorname{SEM}(n=5) .{ }^{*} p<0.05,{ }^{* *} p<0.01$, significantly different when compared to 6-month-old rats. There were no significant differences between 15- and 26-month-old rats. decreased to a higher extent, but significant ROS formation could be still detected. In adult, old and senescent rats, the residual ROS formation was $19.4 \pm 7.1 \%(p<0.001)$, $12.5 \pm 9.8 \%(p<0.001)$ and $9.6 \pm 2.9 \%(p<0.001)$ of the rate in the absence of inhibitors.

To evaluate whether increased ROS production is associated with accumulation of mitochondrial protein damage, we measured total thiol group content and tryptophan degradation. As shown in Fig. 3A, thiol group content decreased in old rats by $8.8 \pm 1.8 \%(p<0.01)$ and in senescent rats by $14.8 \pm 0.6$ $\%(p<0.001)$. Aging resulted in significant degradation of tryptophan (Fig. 3B), compared to adult rats, the fluorescence intensity of tryptophan in proteins decreased in old rats by $32.5 \pm 4.7 \%(p<0.001)$ and in senescent rats by $42.2 \pm 4.3 \%$ $(p<0.001)$. Aging was also associated with gradual accumulation of lipid peroxidation products, as detected by TBARS content. In old rats the TBARS level increased by $29.9 \pm 11.1$ $\%(p<0.05)$ and in senescent rats by $45.3 \pm 5.5 \%(p<0.01)$ when compared to adult rats (Fig. 4).

\section{Discussion}

In this study we have investigated the effect of aging on mitochondrial production of ROS and oxidative damage. The results show that succinate-stimulated ROS formation gradually increased during aging. Both, complex I and complex III inhibitors rotenone and antimycin A as well as their combination inhibited ROS formation significantly but not completely. In accordance with ROS formation, lipid and protein oxidative damage accumulated progressively with age.

Several studies have also shown increased mitochondrial ROS generation during aging but in most of them the potential ROS-generating sites were not tested (Sohal et al. 
1994; Bejma et al. 2000; Moghaddas et al. 2003; Petrosilo et al. 2009). Our results are in agreement with studies on rat hearts, which showed higher ROS production in 24-25month-old rats than in 4-8-month-old ones (Bejma et al. 2000; Moghaddas et al. 2003; Petrosilo et al. 2009). Our study shows that significant increase in ROS formation occurs already in 15-month-old rats. In addition, present study shows that oxidative damage, LPO and tryptophan degradation also accumulates progressively with age.

Using myxothiazol, an inhibitor of ubiquinol-binding site $\left(\mathrm{Q}_{\mathrm{O}}\right)$ of cytochrome $\mathrm{b}$ in complex III, Moghaddas et al. (2003) have shown increased ROS leak from complex III of interfibrillar mitochondria of aged rat hearts. Studies focused on mechanism of mitochondrial ROS generation, but not in relation to aging, have shown that sites of ROS leak in ETC are localized not only in complex III but also in complex I (Fato et al. 2009; Tahara et al. 2009). There are two potential mechanisms by which complex I may generate ROS (for review, see Murphy 2009). In the presence of $\mathrm{NAD}^{+}$-linked substrates like glutamate, malate or pyruvate the ROS release occur during forward electron transfer possibly from the reduced FMN center. At succinate-supported respiration the ROS generation is due to RET from succinate dehydrogenase to complex I, since this path is blocked by rotenone. Although the exact site of ROS formation in complex I during RET remains unclear, RET is thought to be an important source of ROS in some tissues at physiological as well as pathological conditions (Murphy 2009). Complete or marked inhibition of succinate-supported ROS formation by rotenone was observed in brain and heart but not in the liver or skeletal muscle (Votyakova and Reynolds 2001; Liu et al. 2002; Tahara et al. 2009). In agreement with these studies, our data indicate that complex I inhibitor rotenone significantly attenuates succinate-supported ROS formation in rat heart. However, the inhibitory effect of rotenone was independent on age. These results suggest that although RET contributes to generation of ROS in cardiac mitochondria at different ages, it is not responsible for their increased formation in senescence. In contrast to rotenone, the inhibitory effect of antimycin A or combination of antimycin A with rotenone increased with age, indicating that antimycin A-sensitive path may play a role in age-dependent accumulation of ROS. Our results are in agreement with number of studies, which also showed blockage of succinate-supported ROS formation by antimycin A (Votyakova and Reynolds 2001; Kudin et al. 2005; 2008; Tahara et al. 2009), but are in opposite to those showing its stimulatory effect (Raha et al. 2000; Starkov and Fiskum 2001; Liu et al. 2002). Several factors may account for these conflicting results, including differences in tissues/organs, integrity of mitochondria, membrane potential, oxygen tension or concentrations of inhibitors. Moreover, Hansdorf and coworkers (1997) have shown that depending on the presence of $\mathrm{Mn}^{2+}$ ions, antimycin A can either stimu- late or inhibit succinate-supported ROS formation. While stimulatory effect of antimycin A on superoxide production can be explained by accumulation of ubisemiquinone radical in complex III (Raha et al. 2000), specification of its inhibitory effect is less clear, but it indicates leak of ROS from complex I (Kudin et al. 2004). Interestingly, when we apply combination of rotenone and antimycin A, the ROS formation was not completely inhibited. This remaining ROS formation cannot be caused by complex I but is likely due to a leak of ROS from complex III (Votyakova and Reynolds 2001). Thus, these results suggest that both, complex I and III participate in mitochondrial ROS formation, but RET mechanism does not contribute to age-dependent increase. Increased rate of ROS production during aging may be related to altered function of ETC complexes (Gómez and Hagen 2012). Recently, using the same experimental model we have shown age-related loss in activities of ETC complexes (Tatarkova et al. 2011). However, in addition to succinate-stimulated ROS production, tested in the present study, other mitochondrial sites may contribute to ROS leak. In addition to NADH supported respiration which induces ROS leak from complex I by forward mechanism, many other mitochondrial enzymes can produce superoxide radical, including 2-oxoglutarate dehydrogenase and pyruvate dehydrogenase (see Murphy 2009).

In conclusion, our data indicate that various mitochondrial sites can contribute to accelerated ROS generation during aging, including complex I and complex III of ETC. Further studies are needed to localize ROS-producing sites and to identify mechanisms of ROS formation.

The authors declare none conflicts of interest in relation to this article.

Acknowledgements. This work was partially supported by grants VEGA 1/0028/11 from the Ministry of Education and Science of the Slovak Republic and project "Center of Excellence for Research on Personalized Therapy (CEVYPET)", code 2622012053, co-financed from EU sources and European Regional Development Fund.

\section{References}

Babušíková E., Kaplán P., Lehotský J., Jeseňák M., Dobrota D. (2004): Oxidative modification of rat cardiac mitochondrial membranes and myofibrils by hydroxyl radicals. Gen. Physiol. Biophys. 23, 327-335

Barja G. (1999): Mitochondrial oxygen radical generation and leak: sites of production in states 4 and $\mathbf{3}$, organ specificity, and relation to aging and longevity. J. Bioenerg. Biomembr. 31, 347-366 http://dx.doi.org/10.1023/A:1005427919188

Bejma J., Ramires P., Ji L. L. (2000): Free radical generation and oxidative stress with ageing and exercise: differential effects in the myocardium and liver. Acta Physiol. Scand. 169, 343-351 http://dx.doi.org/10.1046/j.1365-201x.2000.00745.x 
Choksi K. B., Papaconstantinou J. (2008): Age-related alterations in oxidatively damaged proteins of mouse heart mitochondrial electron transport chain complexes. Free Radic. Biol. Med. 44, 1795-1805 http://dx.doi.org/10.1016/j.freeradbiomed.2008.01.032

Dai, D.-F., Rabinovitch P. S., Ungvari Z. (2012): Mitochondria and cardiovascular aging. Circ. Res. 110, 1109-1124 http://dx.doi.org/10.1161/CIRCRESAHA.111.246140

Fato R., Bergamini C., Bortolus M., Maniero A. L., Leoni S., Ohnishi T., Lenaz G. (2009): Differential effects of mitochondrial Complex I inhibitors on production of reactive oxygen species. Biochim. Biophys. Acta 1787, 384-392 http://dx.doi.org/10.1016/j.bbabio.2008.11.003

Gómez L. A., Hagen T. M. (2012) Age-related decline in mitochondrial bioenergetics: Does supercomplex destabilization determine lower oxidative capacity and higher superoxide production? Semin. Cell Dev. Biol. 23, 758-767 http://dx.doi.org/10.1016/j.semcdb.2012.04.002

Hansford R. G., Hogue B. A., Mildaziene V. (1997): Dependence of $\mathrm{H} 2 \mathrm{O} 2$ formation by rat heart mitochondria on substrate availability and donor age. J. Bioenerg. Biomembr. 29, 89-95 http://dx.doi.org/10.1023/A:1022420007908

Hunter J. C., Machikas A. M., Korzick D. H. (2012): Agedependent reductions in mitochondrial respiration are exacerbated by calcium in the female rat heart. Gender. Med 9, 197-206 http://dx.doi.org/10.1016/j.genm.2012.04.001

Kaplan P., Jurkovicova D., Babusikova E., Hudecova S., Racay P., Sirova M., Lehotsky J., Drgova A., Dobrota D., Krizanova O. (2007): Effect of aging on the expression of intracellular Ca2+ transport proteins in a rat heart. Mol. Cell. Biochem. 301, 219-226 http://dx.doi.org/10.1007/s11010-007-9414-9

Kudin A. P., Bimpong-Buta N. Y.-B., Vielhaber S., Elger C. E., Kunz W. S. (2004): Characterization of superoxide-producing sites in isolated brain mitochondria. J. Biol. Chem. 279, 4127-4135 http://dx.doi.org/10.1074/jbc.M310341200

Kudin A. P., Debska-Vielhaber G., Kunz W. S. (2005): Characterization of superoxide production sites in isolated rat brain and skeletal muscle mitochondria. Biomed. Pharmacother. 59, 163-168 http://dx.doi.org/10.1016/j.biopha.2005.03.012

Kudin A. P., Malinska D., Kunz W. S. (2008): Sites of generation of reactive oxygen species in homogenates of brain tissue determined with the use of respiratory substrates and inhibitors. Biochim. Biophys. Acta 1777, 689-695 http://dx.doi.org/10.1016/j.bbabio.2008.05.010

Liu Y., Fiskum G., Schubert D. (2002): Generation of reactive oxygen species by the mitochondrial electron transport chain. J. Neurochem. 80, 780-787 http://dx.doi.org/10.1046/j.0022-3042.2002.00744.x

Moghaddas S., Hoppel C. L., Lesnefsky E. J. (2003): Aging defect at the Qo site of complex III augments oxyradical production in rat heart interfibrillar mitochondria. Arch. Biochem. Biophys. 414, 59-66 http://dx.doi.org/10.1016/S0003-9861(03)00166-8
Murphy M. P. (2009): How mitochondria produce reactive oxygen species. Biochem. J. 417, 1-13 http://dx.doi.org/10.1042/BJ20081386

Petrosillo G., Matera M., Moro N., Ruggiero F. M., Paradies G. (2009): Mitochondrial complex I dysfunction in rat heart with aging: critical role of reactive oxygen species and cardiolipin. Free Radic. Biol. Med. 46, 88-94 http://dx.doi.org/10.1016/j.freeradbiomed.2008.09.031

Preston C. C., Oberlin A. S., Holmuhamedov E. L., Gupta A., Sagar S., Syed R. H., Siddiqui S. A., Raghavakaimal S., Terzic A., Jahangir A. (2008): Aging-induced alterations in gene transcripts and functional activity of mitochondrial oxidative phosphorylation complexes in the heart. Mech. Ageing Dev. 129, 304-312 http://dx.doi.org/10.1016/j.mad.2008.02.010

Raha S., McEachern G. E., Myint A. T., Robinson B. H. (2000): Superoxides from mitochondrial complex III: The role of manganese superoxide dismutase. Free Radic. Biol. Med. 29, $170-180$ http://dx.doi.org/10.1016/S0891-5849(00)00338-5

Sohal R. S., Ku H.-H., Agarwal S., Forster M. J., Lal H. (1994): Oxidative damage, mitochondrial oxidant generation and antioxidant defenses during aging and in response to food restriction in the mouse. Mech. Ageing Dev. 74, 121-133 http://dx.doi.org/10.1016/0047-6374(94)90104-X

Starkov A. A., Fiskum G. (2001): Myxothiazol induces H2O2 production from mitochondrial respiratory chain. Biochem. Biophys. Res. Commun. 281, 645-650 http://dx.doi.org/10.1006/bbrc.2001.4409

Tatarková Z., Kuka S., Račay P., Lehotský J., Dobrota D., Mištuna D., Kaplán. (2011): Effects of aging on activities of mitochondrial electron transport chain complexes and oxidative damage in rat heart. Physiol. Res. 60, 281-289

Tatarkova Z., Engler I., Calkovska A., Mokra D., Drgova A., Kuka K., Racay P., Lehotsky J., Dobrota D., Kaplan P. (2012): Effect of normobaric oxygen treatment on oxidative stress and enzyme activities in guinea pig heart. Gen. Physiol. Biophys. 31, 179-184 http://dx.doi.org/10.4149/gpb_2012_020

Tahara E. B., Navarete F. D. T., Kowaltowski A. J. (2009): Tissue-, substrate-, and site-specific characteristics of mitochondrial reactive oxygen species generation. Free Radic. Biol. Med. 46, $1283-1297$

http://dx.doi.org/10.1016/j.freeradbiomed.2009.02.008

Votyakova T.V., Reynolds I.J. (2001): $\Delta \Psi \mathrm{m}$-dependent and -independent production of reactive oxygen species by rat brain mitochondria. J. Neurochem. 79, 266-277 http://dx.doi.org/10.1046/j.1471-4159.2001.00548.x

Wanagat J., Wolf M. R., Aiken J. D. (2002): Age-associated changes in function, structure and mitochondrial genetic and enzymatic abnormalities in the Fischer 344 x Brown Norway F1 hybrid rat heart. J. Mol. Cell. Cardiol. 34, 17-28 http://dx.doi.org/10.1006/jmcc.2001.1483

Received: April 9, 2013

Final version accepted: June 4, 2013 